\title{
Investigation of Polypyrrole Coated Cotton Fabrics as Electromagnetic Shielding Material
}

\author{
Zehra YILDIZ ${ }^{1}$, Ismail USTA ${ }^{1}$, Atilla GUNGOR ${ }^{2}$, Ayşen H. ONEN ${ }^{3}$ \\ ${ }^{\prime}$ Marmara University, Faculty of Technology, Department of Textile Engineering, Istanbul, Turkey \\ ${ }^{2}$ Marmara University, Faculty of Arts and Sciences, Department of Chemistry, Istanbul, Turkey \\ ${ }^{3}$ Istanbul Technical University, Faculty of Arts and Sciences, Department of Chemistry, Istanbul, Turkey
}

\begin{abstract}
This study was aimed to obtain conductive cotton fabric surfaces by chemical oxidative polymerization of pyrrole monomer. For this purpose, iron (III) chloride was used as oxidant, and an aliphatic polyether with acidic groups was used as anionic surfactant respectively. Polypyrrole formation on cotton fabric surface was proved by Fourier transform infrared (FTIR) and scanning electron microscopy (SEM) analysis. The obtained polypyrrole coated cotton fabric samples have been evaluated in terms of electromagnetic shielding effectiveness (EMSE) and electrical conductivity. Results showed that polypyrrole coated cotton fabrics have showed about 25 dB EMSE value in 0-3 $\mathrm{GHz}$ frequency range.
\end{abstract}

Keywords: Polypyrrole, Cotton Fabric, Electromagnetic Shielding, Oxidative Polymerization

\section{INTRODUCTION}

Conductive polymers have superior properties such as low cost, ease of synthesis, flexibility and applicability in so many fields such as light-emitting diodes, electrochromic and electromagnetic shielding materials, anti-static coatings, sensors, energy storage devices etc. Textile fabrics inherently behave as an insulator but can be rendered conductive by various methods such as conductive polymer deposition, conductive particle addition. Recently, conductive polymer coatings have been applied on various textile substrates such as polyester, cotton, wool fabrics etc. [1,2]. In a previous study, a stretchable super capacitor electrode material based on PPy coated cotton knitted fabric has been prepared by electrochemical deposition [3].

In another study, in situ chemical polymerization of pyrrole monomer has been carried out by using ferric chloride as oxidant on cotton, linen, viscose rayon and polyester fabric surfaces. The formation of PPy on textile surfaces has been proven by SEM, FTIR, and XRD analysis [4]. PPy deposition also has been used to obtain antibacterial efficiency in textile materials. The positive charges, that are formed during PPy polymerization, seem to be responsible for the antibacterial activity [5].

In this study, chemical oxidative polymerization of PPy on cotton fabric surfaces was carried out by using an aliphatic polyether with acidic groups as surfactant and ferric chloride as oxidant agent. In chemical oxidative polymerization process, ferric chloride is used as an oxidizing agent helping to oxidize the pyrrole monomer, and forming the chemically active cation radicals. The obtained $\mathrm{Py}+$ cation radicals can dimerize with expulsion of $\mathrm{H}+$. The dimers form cation radicals and react with other monomer molecules, yielding oligomers of insoluble conductive polymers. Cl- ion doped into PPy backbone. Ferric chloride gets reduced ferrous chloride which can be easily removed by water washing. By changing the surfactant percentages, the weight increment, EMSE property and surface electrical resistivity of fabrics have been measured. The formation of PPy on cotton surface was proved by FTIR analysis and SEM images.

\section{MATERIALS AND METHOD}

\subsection{Materials}

Reagent grade pyrrole (Py) monomer was purchased from Sigma-Aldrich. Ferric chloride hexahydrate ( $\mathrm{FeCl} 3.6 \mathrm{H} 2 \mathrm{O})$, and aliphatic polyether with acidic groups (product number= 4242, acid value $=95-105 \mathrm{mgKOH} / \mathrm{g}$ ) were purchased from Merck and DELTA-DC respectively. As textile material 113 $\mathrm{g} / \mathrm{m} 2$ plain weaved cotton fabric ( $30 \mathrm{warp} / \mathrm{cm}, 22 \mathrm{weft} / \mathrm{cm}$ ) was used.

Corresponding Author: Zehra YILDIZ, Tel: +90 21641405 45, E-mail: zehra.yildiz@marmara.edu.tr Submitted: 15 October 2014, Revised: 04 March 2015, Accepted: 01 July 2015 


\subsection{Method}

Fabric samples were cut in $10 \times 10 \mathrm{~cm}$ dimensions. Then each sample was laid out in a glass vessel. Firstly, $30 \mathrm{~mL}$ pyrrole solution (1 $\mathrm{M}$ with distilled water) and surfactant were mixed and added to the glass vessel onto the fabric. All together mixed by a magnetic stirrer for 30 minutes. Then $30 \mathrm{~mL} \mathrm{FeCl}_{3}$ solution $(0.5 \mathrm{M}$ with distilled water) was added to the vessel dropwise in 30 minutes. The polymerization reaction was ended in 2 hours after the colour of the fabric was completely changed from white to black. The fabric sample was washed ethanol, $1 \mathrm{M} \mathrm{HCl}$ solution and distilled water respectively and then dried in open air.

\subsection{Characterization}

The obtained PPy deposited fabric samples were characterized by using Fourier transform infrared (FTIR; Perkin Elmer Spectrum, 100 ATR-FTIR) spectroscopy. And scanning electron microscopy (SEM; JEOL Ltd, JSM5910LV). By using the ASTM D4935-10 standard [6], a Network Analyzer instrument (Rohde\&Schwarz) was used for the measurement of EMSE values of the fabrics. The coaxial transmission line method was used to measure the SE of the specimens and all measurements were carried out in 0-3000 $\mathrm{MHz}$ frequency range. Surface electrical resistivity was measured by using a Keithley 6517A Electrometer/High Resistance Meter instrument according to the ASTM D257-07 standard [7]. Surface resistivity is measured by applying a voltage potential across the surface of the specimen, measuring the resultant current and then performing the following calculation where $\rho$ s is the surface resistivity of the specimen, $\mathrm{V}$ is the applied voltage and $\mathrm{I}$ is the current reading from the instrument (1).

$\rho_{s}=\frac{53.4 V}{I}$ ohms $(\Omega)$

\section{RESULTS AND DISCUSSION}

\subsection{Weight Increment}

Table 1 shows the weight increment values of each sample after polymerization process stemming from the PPy deposition on the fabric surface. Among all samples the highest PPy deposition was obtained with the $7.5 \%$ surfactant usage. In $7.5 \%$ surfactant concentration, the PPy coated fabric sample two times heavier than its raw form. It can be seen that whenever surfactant amount increases the weight increment also increases.
Table 1. Weight increment percentages after PPy deposition on cotton fabric by various surfactant amounts.

\begin{tabular}{cc}
\hline Surfactant (\%) & Weight Increment (\%) \\
1 & 58.9 \\
2.5 & 79.1 \\
5 & 97.1 \\
7.5 & 143.8 \\
\hline
\end{tabular}

\subsection{FTIR Spectroscopy}

Figure 1 shows the $\mathrm{H}$ bonding interaction between the cotton fabric surface and PPy structure. The FTIR spectra of the PPy coated fabric samples with various surfactant ratios was given in Figure 2. Accordingly the characteristic peaks of PPy namely; $\mathrm{C}=\mathrm{O}$ stretching, $\mathrm{C}=\mathrm{C}$ stretching, $\mathrm{C}=\mathrm{N}$ stretching, $\mathrm{C}-\mathrm{N}$ stretching , $\mathrm{C}-\mathrm{H}$ stretching, $\mathrm{C}-\mathrm{H}$ wagging vibration and $\mathrm{N}-\mathrm{H}$ stretching were located at 1720 cm-1, $1550 \mathrm{~cm}-1,1450 \mathrm{~cm}-1,1309 \mathrm{~cm}-1,1040 \mathrm{~cm}-1,787$ $\mathrm{cm}-1$, and $617 \mathrm{~cm}-1$ respectively. The peaks at $1550 \mathrm{~cm}-1$ and $1450 \mathrm{~cm}-1$ are assigned to the pyrrole ring vibrations stemming from the combination of $\mathrm{C}=\mathrm{C}$ and $\mathrm{C}-\mathrm{C}$ stretching vibrations. The bands at 1299 and $1166 \mathrm{~cm}-1$ are associated with the C-N stretching vibrations. The band at $672 \mathrm{~cm}-1$ is corresponding to the doped $\mathrm{Cl}$ - ion on the PPy backbone.

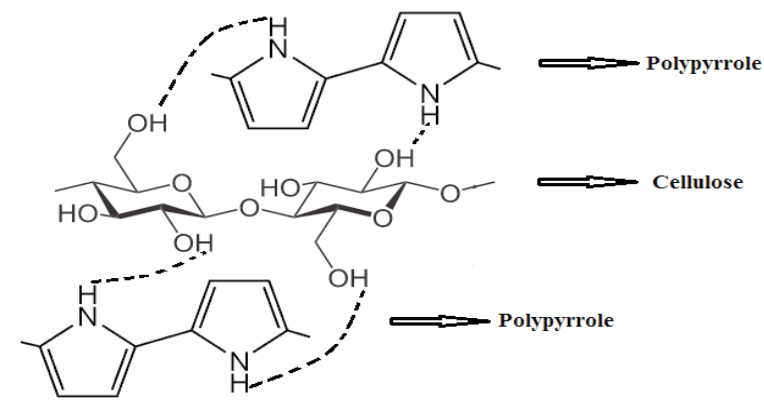

Figure 1. Illustration of $\mathrm{H}$ bonding between cotton surface and polypyrrole.

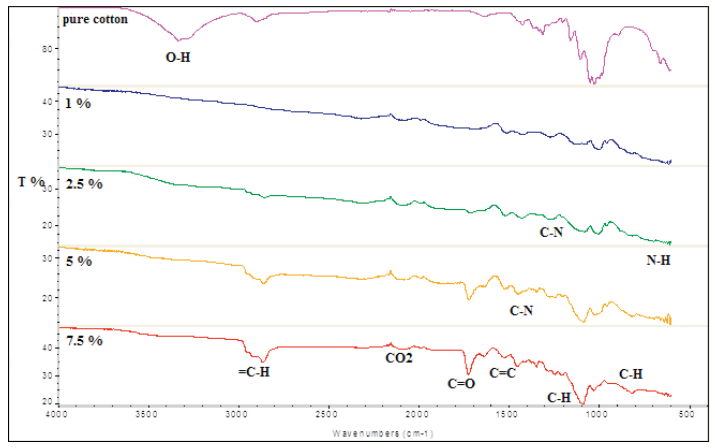

Figure 2. FTIR spectra of pure cotton and PPy coated cotton fabrics by various surfactant percentages. 


\subsection{SEM Analysis}

Figure 3 shows the SEM images of pure cotton fabric and fabrics containing 2.5 and $7.5 \%$ surfactant. Accordingly, when the surfactant amount was set as $2.5 \%$, the PPy completely coated the fabric surface. But in $7.5 \%$ surfactant addition, there are some agglomeration on the fabric surface has been observed.

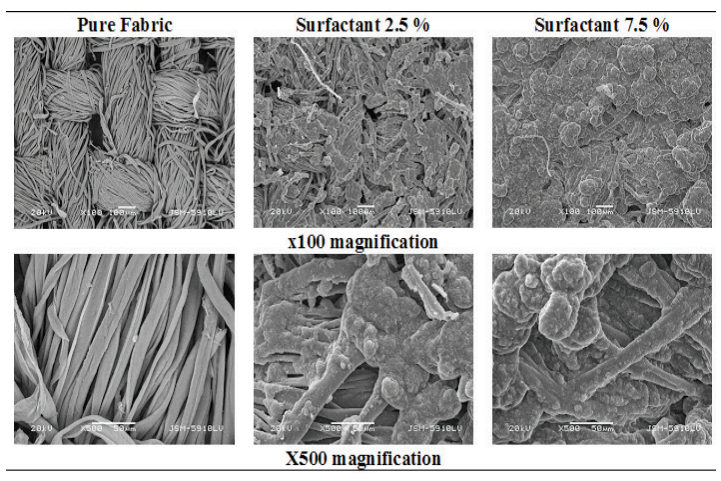

Figure 3. SEM images of the pure fabric and fabrics with 2.5 $\%$ and $7.5 \%$ surfactant addition in various magnifications.

\subsection{EMSE Measurement}

The EMSE values of the PPy coated cotton fabric samples with various surfactant percentages were given in Figure 4. Accordingly, the highest shielding value was obtained as about $24 \mathrm{~dB}$ at $2280 \mathrm{MHz}$ when the surfactant percentage was taken as $2.5 \%$. In general all samples showed the highest shielding effect in $2000-2500 \mathrm{MHz}$ frequency range. Considering the shielding efficiency, $2.5 \%$ surfactant amount can be accepted as the optimum surfactant concentration. After that point increment in surfactant amount causes decline in shielding property. This result is stemming from the excess $\mathrm{Fe}$ ions that are not participating to the polymerization process. Furthermore, the excess Fe ions cause over-oxidation of the yarn that leads the formation of carbonyl defects on the PPy backbone. Carbonyl defects cause interruptions on the $\square$ conjugation and act as electron withdrawing groups, inhibiting charge transfer, resulting decline in conductivity [8-10].

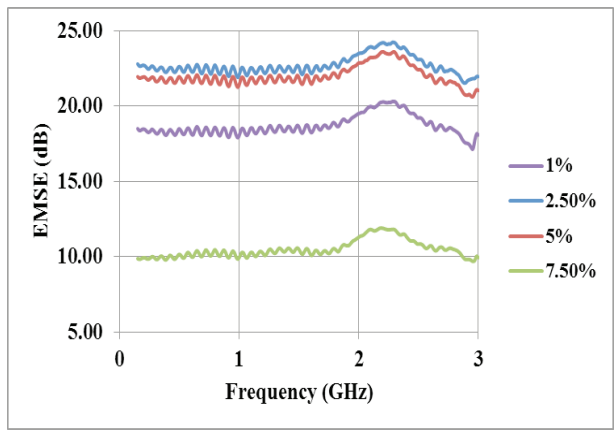

Figure 4. EMSE values of PPy coated cotton fabrics with various surfactant amounts.
Table 2 shows the shielding effectiveness (SE), absorption (A), reflection (R) and transmission (T) values of the PPy deposited cotton fabrics with various surfactant concentrations. The frequencies of 1005, 1500, 1800 and $2505 \mathrm{MHz}$ were chosen because these are the mostly exposed frequencies especially emitting from electronic devices, mobile phones and radio frequencies etc. Accordingly, the highest shielding value was obtained in the $2.5 \%$ surfactant concentration. In all samples, the electromagnetic shielding property shows an absorbance dominant property instead of reflection because conductive polymers have an absorption dominant shielding property [11].

Table 2. Shielding effectiveness (SE), absorption (A), reflection $(\mathrm{R})$ and transmission $(\mathrm{T})$ values of the PPy deposited cotton fabrics with various surfactant concentrations.

\begin{tabular}{|c|c|c|c|c|c|c|c|c|c|c|c|c|c|c|c|c|}
\hline \multirow{3}{*}{$\begin{array}{c}\text { Surfactant } \\
\text { Concentration } \\
(\%)\end{array}$} & \multicolumn{16}{|c|}{ Frequency $(\mathrm{MHz})$} \\
\hline & \multicolumn{4}{|c|}{1005} & \multicolumn{4}{|c|}{1500} & \multicolumn{4}{|c|}{1800} & \multicolumn{4}{|c|}{2505} \\
\hline & $\begin{array}{c}\mathrm{SE} \\
(\mathrm{dB}) \\
\end{array}$ & A & R & $\mathrm{T}$ & $\begin{array}{c}S E \\
(\mathrm{~dB}) \\
\end{array}$ & A & $\mathrm{R}$ & $\mathrm{T}$ & $\begin{array}{c}\mathrm{SE} \\
(\mathrm{dB})\end{array}$ & A & $\mathrm{R}$ & $\mathrm{T}$ & $\begin{array}{c}\mathrm{SE} \\
(\mathrm{dB}) \\
\end{array}$ & $\mathrm{A}$ & $\mathrm{R}$ & $\mathrm{T}$ \\
\hline 1 & 18.19 & 0.51 & & & 18.61 & 0.39 & 0.60 & & 18.37 & 0.63 & 0.36 & 0.01 & 19.220 & 0.740 & 0.25 & 0.01 \\
\hline 2.5 & 22.22 & 0.50 & 0.49 & 0.01 & 22.59 & 0.37 & 0.62 & 0.01 & 22.84 & & 0.37 & 0.01 & 23.090 & & 0.26 & 0.01 \\
\hline 5 & 21.57 & 0.51 & & 0.01 & 21.87 & 0.38 & 0.61 & & 22.16 & & 0.36 & 0.01 & 22.420 & & 0.25 & 0.01 \\
\hline 7.5 & 10.05 & 0.72 & 0.18 & 0.10 & 10.49 & 0.63 & 0.28 & $0 \cap 0$ & 10.53 & 0.81 & 0.11 & 0.08 & 10.860 & 0.840 & 0.08 & 0.08 \\
\hline
\end{tabular}

\subsection{Surface Electrical Resistivity}

Table 3 shows the surface electrical resistivity of the PPy coated cotton fabrics with various surfactant amounts. Accordingly, the least surface electrical resistivity in other words the best electrical conductivity was obtained as $2.39 \times 103 \Omega / \mathrm{sq}$ when $2.5 \%$ surfactant is used. With increasing surfactant amount, surface electrical resistivity also increases.

Table 3. Surface electrical resistivity values of the PPy deposited cotton fabrics with various surfactant concentrations.

Surfactant (\%)
Pure Fabric
1
2.5
5
7.5

Surface Electrical Resistivity $(\Omega /$ sq)

$1.68 \times 10^{5}$

$2.82 \times 10^{3}$

$2.39 \times 10^{3}$

$2.52 \times 10^{3}$

$3.63 \times 10^{3}$ 


\section{CONCLUSSION}

In this study, chemical oxidative polymerization of pyrrole monomer was performed on cotton fabric surfaces by using an anionic surfactant and oxidant $\left(\mathrm{FeCl}_{3}\right)$. By changing the surfactant amount the electromagnetic shielding efficiency and surface electrical resistivity of the PPy coated fabric samples were investigated. PPy formation on fabric samples was proven by FTIR analysis.

Results showed that whenever surfactant amount increases weight uptake of the fabric samples increase. FTIR spectra showed characteristic PPy peaks at $1550 \mathrm{~cm}-1 \quad(\mathrm{C}=\mathrm{C})$, $1450 \mathrm{~cm}-1$ (C-C) and $1309 \mathrm{~cm}-1$ (C-N) respectively. SEM images proved the existence of agglomeration on $7.5 \%$ surfactant added sample. The highest EMSE value with 24 $\mathrm{dB}$ at $2280 \mathrm{MHz}$ was obtained by using $2.5 \%$ surfactant amount. Also the least surface electrical resistivity thus the highest conductivity was obtained at this concentration. In conclusion, the $2.5 \%$ surfactant addition can be accepted as the optimum concentration for this research.

\section{REFERENCES}

[1] G. Liang, L. Zhu, J. Xu, D. Fang, Z. Bai, and W. Xu, Electrochimica Acta, vol. 103, pp. 9-14, (2013).

[2] M. Yanilmaz and A. S. Sarac, Textile Research Journal, p. 0040517513495943, (2014).
[3] B. Yue, C. Wang, X. Ding, and G. G. Wallace, Australian Institute for Innovative Materials-Papers, pp. 17-22, (2013).

[4] K. Firoz Babu, S. Siva Subramanian, and M. Anbu Kulandainathan, Carbohydrate polymers, vol. 94, pp. 487-495, (2013).

[5] A. Varesano, C. Vineis, A. Aluigi, F. Rombaldoni, C. Tonetti, and G. Mazzuchetti, vol. 14, pp. 36-42, (2013).

[6] "ASTM D4935-10: Standard Test Method for Measuring the Electromagnetic Shielding Effectiveness of Planar Materials, p.10,” (2010).

[7] “ASTM D257-07: Standard Test Methods for DC Resistance or Conductance of Insulating Materials, p.18," (2007).

[8] A. Kaynak and R. Beltran, Polymer international, vol. 52, pp. 1021-1026, (2003).

[9] A. Kaynak, L. Wang, C. Hurren, and X. Wang, Fibers and Polymers, vol. 3, pp. 24-30, (2002).

[10] Z. Yildiz, I. Usta, and A. Gungor, Textile Research Journal, October 8, 2012 (2012).

[11] Y. Hong, C. Lee, C. Jeong, J. Sim, K. Kim, J. Joo, M. Kim, J. Lee, S. Jeong, and S. Byun, Current Applied Physics, vol. 1, pp. 439-442, (2001). 